\title{
Mechanical Beam Steering Circular Patch Antenna
}

\author{
I.F. da Costa $^{1}$, H.R.D. Filgueiras ${ }^{1}$, J.R. Kelly ${ }^{2}$, Arismar Cerqueira S. Jr. ${ }^{1}$ and Pei Xiao ${ }^{2}$ \\ ${ }^{1}$ Laboratory WOCA, National Institute of Telecommunications (Inatel), Santa Rita do Sapucaí, Brazil. \\ ${ }^{2}$ ICS/5GIC, The University of Surrey, Stag Hill Campus, Guildford, GU2 7XH, UK. \\ igorf@mtel.inatel.br, arismar@inatel.br
}

\begin{abstract}
This work reports preliminary results and a prototype of an innovative mechanical beam steering circular patch antenna for 5G indoor cellular access networks with sub$6 \mathrm{GHz}$ operation. The beam steering is achieved using 18 screws installed around the circular patch radiator, working as a reflector by proper managing the screws position and height. The new antenna can steer its main beam over $360^{\circ}$ in azimuth plane and from $-30^{\circ}$ to $30^{\circ}$ in the elevation plane with gain up to 8.01 dBi at 4.6 GHz.
\end{abstract}

Index Terms-Antenna, beam steering, circular patch and mechanical reconfiguration.

\section{INTRODUCTION}

The fifth-generation of cellular networks (5G) can become a key evolutionary technology to ensure the viability of the emerging new applications, such as Internet of Things (IoT), wearable devices and autonomous cars. Spectrum for $5 \mathrm{G}$ services is typically considered in two categories: sub-6 $\mathrm{GHz}$ frequencies, which include the traditional cellular bands, for low latency and large coverage applications; and above 6 $\mathrm{GHz}$, which includes the mm-waves bands, for ultrawideband applications [1]. Both low-band and high-band frequencies will be combined into a common system architecture, with devices able to connect to both accesses simultaneously.

With such new applications, the wireless environment tends to become multitask and extremely dynamic. In this context, reconfigurable antennas can be considered as potential solutions to guarantee uniform and continuous coverage and connectivity [2, 3]. An antenna reconfiguration is achieved by changing the radiated fields. It is basically a remodeling in the antenna current and/or in its radiation element [4]. The reconfiguration ability makes an antenna able to operate in many wireless applications, such as mobile networks, find and track applications, to name a few [5].

The reconfiguration technique has to be defined at the beginning of the antenna design, as well as the antenna requirements. There are basically four reconfiguration types [6]: bandwidth reconfiguration; polarization reconfiguration; radiation pattern reconfiguration; or a combination of any of these characteristics. Technically speaking, the reconfiguration techniques can be classified into four major categories [7]: electrical; mechanical; optical; material. Particularly, we have been working on the development of reconfigurable antennas for mm-waves applications. In [8], we proposed an optically-controlled slotted-waveguide antenna array able to reconfigure its bandwidth between 28 and $38 \mathrm{GHz}$ bands, by properly illuminating a photoconductive switch. Moreover, a mechanically reconfigurable slotted-waveguide antenna array capable to provide radiation pattern reconfiguration was described in [9].

One of the most precise beam steering techniques is the phased array antennas [10]. Planar structures with different phase per elements gives the possibility to reconfigure the radiation pattern in both azimuth and elevation planes. The number of antennas defines the angle precision. In order to enhance the scanning angle, a large number of elements is required, significantly increasing the operational costs.

In this paper, we present a screw-based beam steering technique applied to a reconfigurable circular patch antenna. It consists of a circular element surrounded by 18 screws, acting as a dynamic reflector in order to reconfigure the antenna radiation pattern. Fig. 1 illustrates a possible application of the proposed antenna in sub- $6 \mathrm{GHz} 5 \mathrm{G}$ network for autonomous car applications.

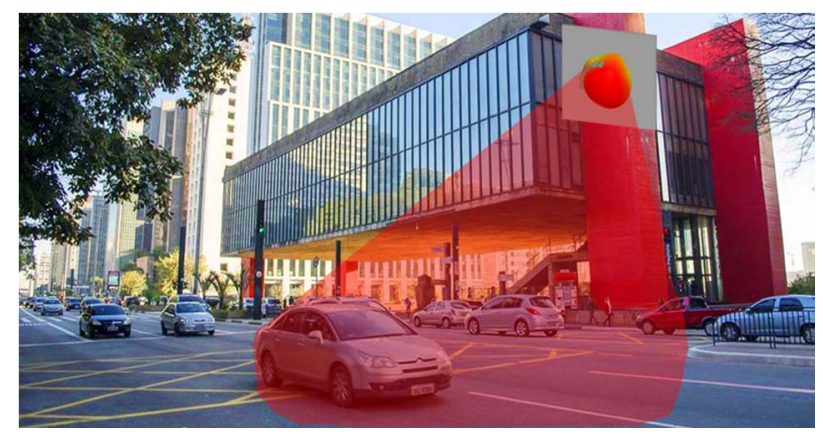

Fig. 1. Sub-6 GHz 5G network for autonomous car applications.

The manuscript is divided in other three sections. Section II presents the antenna design and reconfiguration concept. The numerical results and proof of concept are presented in Section III. Finally in Section IV we discuss our conclusions and propose future works.

\section{ANTENNA DESIGN AND RECONFIGURATION TECHNICHE}

The proposed structure is composed by only one radiating element with some screws around it, working as a reflector. Fig. 2 shows the proposed structure with 18 screws and an $11.85 \mathrm{~mm}$ radius circular patch. The patch is printed on an Arlon DiClad 880 substrate with thickness of $1.58 \mathrm{~mm}$ and dielectric constant of 2.2. The ground plane is defined by $\mathrm{x}=$ $73.32 \mathrm{~mm}$ and $\mathrm{y}=73.32 \mathrm{~mm}$, ensuring approximately half- 
wavelength at $4.6 \mathrm{GHz}$ from each screw to the substrate edges. The antenna design parameters have been optimized using ANSYS HFSS; its final dimensions are: $r=11.85 \mathrm{~mm}, \mathrm{~d}=4$ $\mathrm{mm}$ and $\mathrm{r}_{\mathrm{s}}=15 \mathrm{~mm}$. The feeding point was shifted from the center of the circular patch by $3.5 \mathrm{~mm}$ in the $x$-axis and excited by a coaxial cable.

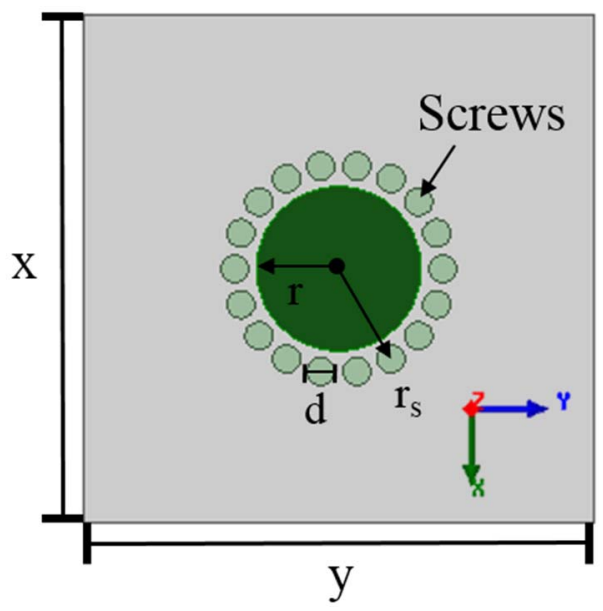

Fig. 2: Mechanical beam steering circular patch antenna.

The antenna radiation pattern is mechanically reconfigured by means of screw and unscrew the pins from the structure bottom to top in order to steer the beam in both azimuth and elevation planes. This technique has a potential to provide the same precision of phased antenna arrays with the remarkable advantages of lower side lobe level and significant cost reduction. Fig. 3 points up an example of the reconfiguration mechanism. Six screws have been used in the configuration illustrated in Fig. 3a for pointing the antenna main beam to $\left(\phi=0^{\circ}\right)$ in the azimuth plane $(x y)$. Subsequently, a different six screws configuration have been applied in order to ensure a beam steering at $\left(\phi=-45^{\circ}\right)$, as shown in Fig. $3 b$. By continuing gradually managing the screws, one can steer the main beam over the entire azimuth plane. Moreover, the number of screws to be twisted in can manipulate the halfpower beamwidth.

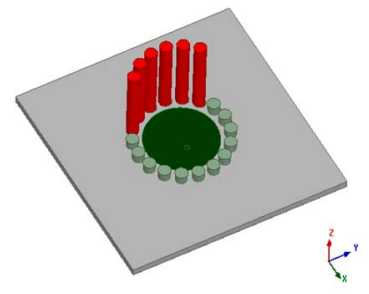

a) Configuration 1: pointing at $\phi=0^{\circ}$.

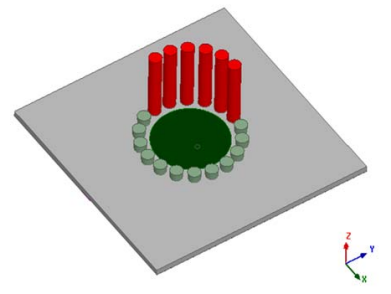

b) Configuration 2: pointing at $\phi=$ $-45^{\circ}$.
Fig. 3. Mechanical beam steering illustration in the azimuth plane.

Our technique provides two degrees of freedom for the radiation pattern reconfiguration: the screw position and their height from the substrate. The screw position will define the steering angle in the azimuth plane $(\phi)$, while the screw height allows to change the steering angle in the elevation plane- $x z$ $(\theta)$.

\section{NUMERICAL RESULTS AND PROTOTYPE}

This section reports the mechanical beam steering antenna simulation results and its prototype. ANSYS HFSS has been used to elaborate the antenna numerical model and optimize its performance. In order to analyze the beam steering in the azimuth and elevation planes, we had elaborated two different scenarios:

1. Scenario I: Configuration 1 (pointing at $\phi=0^{\circ}$ ) and sweep of the screw height from 1 to $20 \mathrm{~mm}$.

2. Scenario II: Screw height of $6 \mathrm{~mm}$ and screw position for pointing at $\phi=75^{\circ}$ and $\phi=-105^{\circ}$.

The Scenario I reflection coefficient $\left(\mathrm{S}_{11}\right)$ is displayed in Fig. 4 and the antenna bandwidth is defined as the frequency range in which $\mathrm{S}_{11}$ is below $-10 \mathrm{~dB}$. The antenna input impedance experiences a small alteration with the screw height, since its resonant frequency is shifted by $2.12 \%$ and its bandwidth varies from $3 \%$ and $4.89 \%$, as the height is increased from 0 (screw on the substrate level) to $20 \mathrm{~mm}$. As a resume, the antenna bandwidth is from 4.54 to $4.68 \mathrm{GHz}$ for $19 \mathrm{~mm}$ height, 4.54 to $4.77 \mathrm{GHz}$ for $10 \mathrm{~mm}$ height and from 4.53 to $4.73 \mathrm{GHz}$ for $20 \mathrm{~mm}$ height, giving rise to common bandwidth, which satisfies all height variations, of $3.03 \%$ from 4.54 to $4.68 \mathrm{GHz}$.

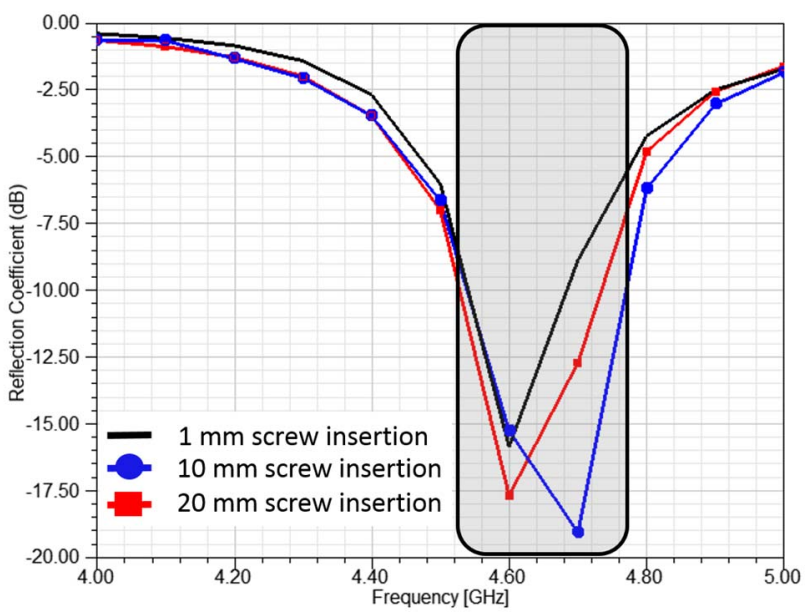

Fig.4. Reflection coefficient as a function of screw height.

The main objective of the Scenario II is to analyze the beam steering in the elevation plane as a function of the screw height. Fig. 5 depicts the simulated radiation pattern results at 4.6 $\mathrm{GHz}$, demonstrating that the mechanical reconfiguration enables to smoothly steer the radiation pattern from 0 to $30^{\circ}$ in the elevation plane. The gain varies from 7.9 to $8.01 \mathrm{dBi}$ with the screw height variation, giving rise a gain flatness of $0.11 \mathrm{~dB}$. The steering step only depends on the stepper motors accuracy. The smaller the motor rotation step is, better the beam control will be. 


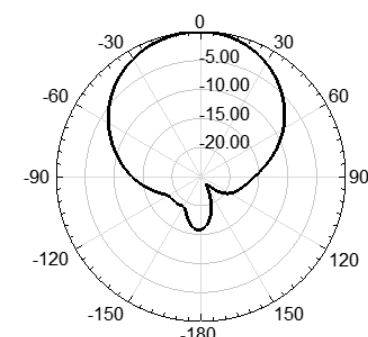

a) $0 \mathrm{~mm}$ (screw on the substrate level).

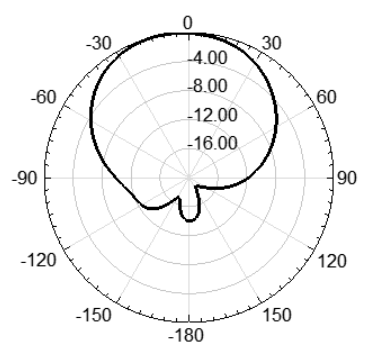

b) $10 \mathrm{~mm}$.

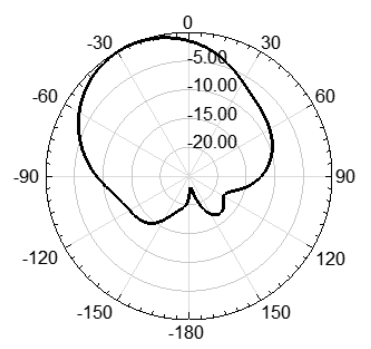

c) $20 \mathrm{~mm}$.

Fig. 5. Elevation plane beam steering as a function of screw height.

The Scenario II also aims to illustrate the beam steering capability in the azimuth plane, by using six screws at $6 \mathrm{~mm}$ height. Fig. 6 presents the obtained reflection coefficient results for this scenario. Once again, the antenna input impedance was slightly varied, as a consequence of the mechanical reconfiguration: bandwidth of $4.56 \%$ centered at 4.6 GHz for the first case; $3.61 \%$ bandwidth at $4.7 \mathrm{GHz}$ for the second case. This variation comes from the distance from the group of screws to the antenna feeding point, since all screws are in the reactive near-field region. The common bandwidth, which satisfies both cases, is from 4.6 to 4.73 $\mathrm{GHz}$, performing a fractional bandwidth of $2.79 \%$.

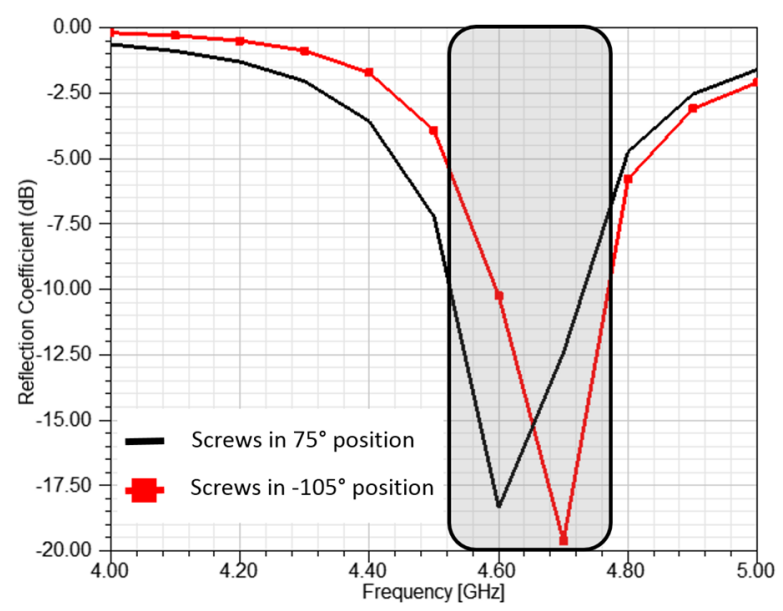

Fig.6. Reflection coefficient as a function of the screw position.

Figs. $7 \mathrm{a}$ and $7 \mathrm{~b}$ reports other possible beam steering configurations at $4.6 \mathrm{GHz}$ in the azimuth and elevation planes, with the aim of demonstrating the applicability of the proposed mechanical beam steering circular patch antenna.
As a conclusion, the proposed antenna makes possible to entirely cover the azimuth plane and from $-30^{\circ}$ to $30^{\circ}$ in the elevation plane. Finally, Fig. 8 reports a photograph of the proposed antenna prototype, which is going to be experimentally characterized in terms of reflection coefficient and radiation pattern.

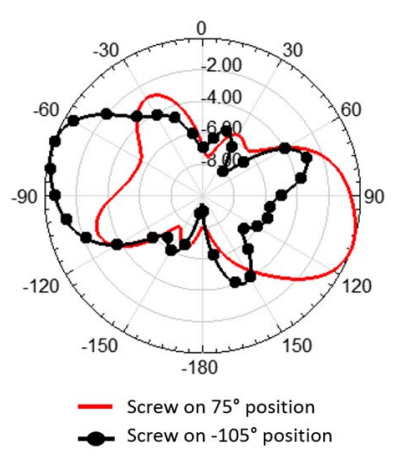

a) Azimuth plane

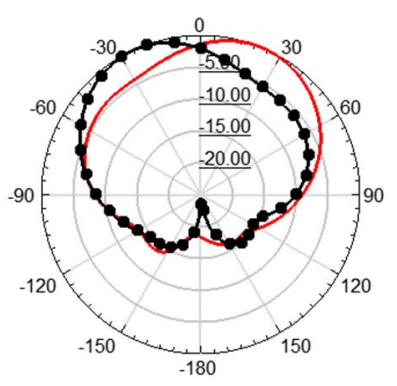

- Screw on $75^{\circ}$ position

- Screw on $-105^{\circ}$ position

b) Elevation plane
Fig.7. Demonstration of beam steering capability in the azimuth and elevation planes.
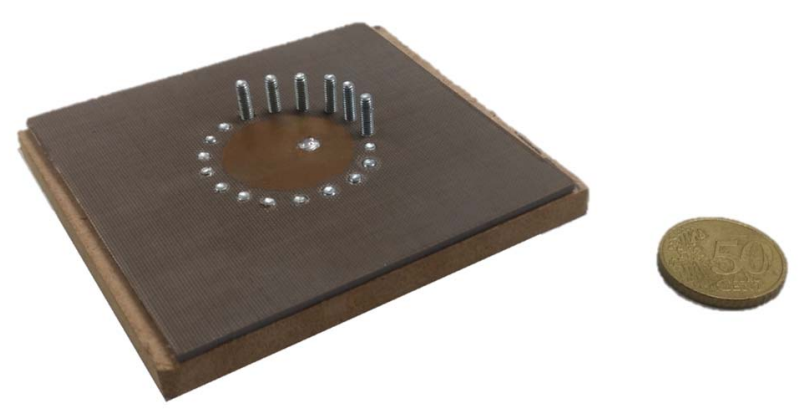

Fig.8. The mechanical beam steering circular patch antenna prototype.

\section{CONCLUSIONS}

We have introduced the concept and reported numerical preliminary results and a prototype of a mechanical beam steering circular patch antenna. The proposed antenna is based on a unique radiating element and a series of screws surrounding it and working as a reconfigurable reflector. Managing the screw position and height enables the beam steering capability. Its potentiality has been demonstrated by numerical simulations of the antenna radiation pattern in the azimuth and elevation planes at $4.6 \mathrm{GHz}$.

The antenna input impedance has been slightly influenced by the reconfiguration mechanism, requiring a definition of a common bandwidth, which satisfies all beam steering configurations. For this reason, the antenna bandwidth varied from 2.79 to $3.03 \%$, depending on the plane that demands beam steering. Additionally, the proposed antenna makes possible to perform beam steering entirely covering the azimuth plane and from $-30^{\circ}$ to $30^{\circ}$ in the elevation plane. Its gain varied from 7.9 to $8.01 \mathrm{dBi}$, giving rise a gain flatness of only $0.11 \mathrm{~dB}$. All those properties make it a viable solution 
for future wireless networks, since it provides the same precision of phased antenna arrays with significant cost reduction.

As a future work, we aim to experimentally characterized the antenna prototype in terms of reflection coefficient and radiation pattern. Furthermore, it is aimed to scale the proposed antenna to mm-waves and implement its both versions in a real dual-band $5 \mathrm{G}$ network.

\section{ACKNOWLEDGMENTS}

This work was partially supported by Finep/Funttel Grant No. 01.14.0231.00, under the Radio Communications Reference Centre (CRR) project of the National Institute of Telecommunications (Inatel), Brazil. Authors also thank the financial support from CNPq, CAPES, MCTI and FAPEMIG and technical support from Keysight and ESSS-ANSYS. We also would like to acknowledge the support of the University of Surrey 5GIC (http://www.surrey.ac.uk/5gic) members for this work.

\section{REFERENCES}

[1] W. H. Chin, Z. Fan and R. Haines, "Emerging technologies and research challenges for $5 \mathrm{G}$ wireless networks," in IEEE Wireless Communications, vol. 21, no. 2, pp. 106-112, April 2014.

[2] A. I. Sulyman, A. Alwarafy, G. R. MacCartney, T. S. Rappaport and A. Alsanie, "Directional Radio Propagation Path Loss Models for Millimeter Wave Wireless Networks in the 28-, 60-, and 73-GHz Bands," in IEEE Transactions on Wireless Communications, vol. 15, no. 10.

[3] Rappaport, T.S.; Shu Sun; Mayzus, R.; Hang Zhao; Azar, Y.; Wang, K.; Wong, G.N.; Schulz, J.K.; Samimi, M.; Gutierrez, F., "Millimeter Wave Mobile Communications for 5G Cellular: It Will Work!," Access, IEEE, vol.1, no., pp.335,349, 2013.

[4] 6939-6947, Oct. 2016. J. Costantine, Y. Tawk, and C. G. Christodoulou, "Design of Reconfigurable Antennas Using Graph Models", San Rafael, CA, USA: Morgan and Claypool, 2013.

[5] C. G. Christodoulou, Y. Tawk, S. A. Lane, and S. R. Erwin, "Reconfigurable antennas for wireless and space applications", Proceedings of the IEEE, vol. 100, no. 7, pp. 2250-2261, April, 2012.

[6] J. Costantine, Y. Tawk, S. E. Barbin, C. G. Chritodoulou, "Reconfigurable Antennas: Design and Ampplications", Proceedings of the IEE, vol. 3, No. 3, March, 2015.

[7] J. T. Bernhard, Reconfigurable Antennas.San Rafael, CA, USA: Morgan and Claypool, 2007.

[8] I. F. da Costa, Arismar Cerqueira S. Jr, D. H. Spadoti, L. G. da Silva, J. A. J. Ribeiro, S. E. Barbin, "Optically Controlled Reconfigurable Antenna Array for mm-wave Applications", IEEE Antennas and Wireless Propagation Letters (AWPL), vol. 16, pp. 2142-2145, May 02, 2017.

[9] H.R.D. Filgueiras, I. F. da Costa, R. A. Santos, James R. Kelly, Arismar Cerqueira S. Jr., "Mechanically Reconfigurable Slotted-Waveguide Antenna Array for 5G Networks", International Microwave and Optoelectronics Conference (IMOC), 2017.

[10] I. Uchendu, James R. Kelly, "Combined Parasitic and Phased Array Reconfigurable Antenna", in Loughborough Antennas \& Propagation Conference (LAPC), 2015 Loughbotough, 2015. 